\title{
I7. COMMISSION DU MOUVEMENT ET DE LA FIGURE DE LA LUNE
}

Président: M. C. B. Watts.

Membres: MM. Banachiewicz $\dagger$, Boneff, Brouwer, Eckert, Guth, Hirose, Koziel, Mme McBain Sadler, MM. Markowitz, Meyer, Nefediev, Ueta, Weimer, Yakovkin.

We note with much regret the death on I7 November I954 of Prof. T. Banachiewicz, formerly President of this commission.

\section{Progress of Research}

Investigations of the rotation elements and of the figure of the Moon have been continued in the U.S.S.R. At the Engelhardt Observatory, Kasan, A. A. Nefediev continued his heliometer measures and made yo observations of Mösting A. S. T. Habibulin obtained II5 photographs of the Moon with a horizontal telescope of $8 \mathrm{~m}$. focal length. At Kiev A. K. Ossipov obtained I69 photographs at the University Observatory, and I. V. Gavrylov, at the Central Observatory of the Ukrainian Academy of Sciences, began a series of photographic observations, pairs of stars being used for scale.

Nefediev completed the reduction of the early heliometer measures made by Krassnov at Kasan, r895-98. He obtained for the mean inclination of the equator and the function $f$ of the moments about the principal axes, $I=\mathrm{I}^{\circ} 32^{\prime} \mathrm{IO}^{\prime \prime} \pm \mathrm{I} 8^{\prime \prime}$ and $f=0.63 \pm 0.05$ respectively. After the second approximation he obtained for $f, 0.60$ and 0.7 I. Treating the apparent radius of the Moon as a function of the libration in latitude he obtained

$$
\Delta h=-\mathrm{I}^{\prime \prime} \cdot 38 \pm 0 \text { "05 }+\left(0 " 057 \pm 0 \text { "or3) } \beta_{0}\right. \text {. }
$$

Using the cracovian method, Nefediev re-reduced his own observations, obtaining $I=\mathrm{r}^{\circ} 33^{\prime} 26^{\prime \prime} \pm 20^{\prime \prime}$ and $f=0.68 \pm 0.04$. The second approximation gave $f=0.7 \mathrm{I}$ and $0.6 \mathrm{r}$.

The effect of the libration in latitude on the apparent radius was negligible $\left(+0\right.$ "oog $\left.\beta_{0}\right)$ in the results of a joint reduction of measures involving both east and west limbs; but in separate reductions the coefficient of $\beta_{0}$ appears as $-00^{\prime \prime} 025$ and $+00^{\prime \prime} 035$ for the east and west limbs respectively.

Yakovkin has re-reduced observations made by Banachiewicz during the period I9I0-I5, taking into account the relation between radius and libration, and obtains $I=\mathrm{I}^{\circ} 32^{\prime} 49^{\prime \prime} \pm \mathrm{I} 2^{\prime \prime}$ and $f=0.85 \pm 0.03$

Habibulin has developed a new method of determining $f$, using the amplitude of the largest term in the physical libration in longitude. From observations of Banachiewicz, Belkovich, and Michailovsky he finds $f=0 \cdot 60$. He is continuing the re-reduction of observations of Krassnov, Yakovkin, and Nefediev.

At the Paris Observatory Weimer has completed his Atlas de profils lunaires with the publication of the second part, consisting of 72 profiles of the west limb of the Moon. In a recent memoir (a thesis presented to the Faculty of Science of the University of Paris), he discusses the methods used in the production of the profiles as well as some related subjects. Among these are the change in the aspect of the limb as a function of the librations and practical definitions of a suitable circle of reference. He illustrates with some examples the improvement in the results of occultations when corrected with data taken from his profiles.

The circle to which the elevations of Weimer's profiles are referred is admittedly a first approximation and he considers that it may be practicable to obtain a second approximation by the use of occultations.

Dr G. Schrutka-Rechtenstamm, at the University Observatory, Vienna, has discussed the problems arising in the derivation of the rotation elements from heliometer measures. $\mathrm{He}$ has found an alternative method of treating the difficulty in determining the value of $f$ in the neighbourhood of the critical value, 0.662 . The method involves the introduction of the coefficient of $\sin 2 \omega$ as a sixth unknown. 
Large-scale limb irregularities no doubt have seriously affected the accuracy of investigations of the Moon's rotation elements. It is certain that definitive limb corrections would help greatly in this problem. Yakovkin stresses the importance of having such corrections before a general reduction of the heliometer measures of the past $\mathrm{r}_{50}$ years is undertaken.

In connexion with his survey of the marginal zone, Watts is examining a method of determining the inclination of the Moon's equator which is insensitive to limb irregularities. It involves the use of precise measures in position angle on photographs of the Moon. The photographs used, being provided with orientation markings, make the following procedure possible:

On two photographs, exposed on widely separated occasions, but at nearly the same librations and phase, a well-defined limb feature near the intersection of the two limb lines is selected. Its position angle from the north is then measured, using as origin in each case the centre of the circular arc that best fits the bright limb. By application of the computed position angle $C$ of the Moon's axis for the respective dates, the measures are referred to the axis. These measures are, of course, subject to a small error originating in the displacement of the origin from the centre of mass, but it should be nearly the same in both photographs on account of the similarity in the librations. The difference between the measures therefore will be almost free from this error. When it has been corrected for the lack of coincidence in the librations, the difference can be used in an observation equation in which the unknowns are a correction to the value of $I$ used in computing $C$ and terms in the physical libration in latitude. The method does not involve the physical libration in longitude and, to be most useful, would have to be used in conjunction with the classical method. This investigation, utilizing about roo photographs, will be completed in 1955 .

At Sofia Prof. Boneff has continued an earlier examination of the arrangement of formations on the Moon which had indicated that they are not distributed at random. From a statistical analysis of the distribution of the larger craters with respect to the mean centre of the disk, and on the hypothesis that they were caused in general by volcanic forces, he concludes that the gravitational attraction of the Earth may also have had some influence in their formation.

At the Tokyo Astronomical Observatory H. Hirose reports that photo-electric observations of occultations have been made during the past three years. These impersonal observations are found to be in good agreement with visual observations made in Japan. From nine sets of occultations observed photo-electrically Hirose derives for the Earth's equatorial radius $6377879 \pm 357 \mathrm{~m}$. With $\mathrm{R}$. Manabe, using occultations of Pleiades stars with Hayn's limb corrections, he derives for the Moon's semi-diameter $932 " 80 \pm 0 " 07$.

Kuiper has recently discussed the figure of the Moon and the origin of its surface features in the Proceedings of the National Academy of Sciences. He has made preparations at the Yerkes Observatory to investigate the physical librations by means of photographic observations.

The British Nautical Almanac Office has continued to predict occultations for about seventy stations. The discussions of reduced observations have been published through I950 in the Astronomical Journal and Mrs McBain Sadler reports that those for I95I-52 are being prepared for publication. The observations have been reduced without limb corrections; but, in anticipation of a re-discussion of observations beginning with I943, the librations have been computed for the application of limb corrections when the survey by Watts is completed. It is intended to include the many observations since I943 received too late for the annual discussions. A combined discussion of the observations for 1943-47 was published as an Appendix to Greenwich Observations 1939, and a similar one for $1948-52$ is being prepared.

The dual-rate Moon position camera developed by Markowitz at the Naval Observatory, Washington, has been in regular use since June I952 ( $A$ str. $J .59,69$, I954). This method of determining the Moon's position possesses the marked advantage of making possible a reference to a large number of points on the Moon's bright limb corresponding 
to one instant. The observer is, of course, not limited to a single observation on a particular night, as is the case with the transit circle. In practice about thirty points and a pair of plates are used. The external probable error in either co-ordinate for two plates is $0 " \mathrm{I} 5$. This should be reduced when limb corrections are applied. It is expected that the method will be utilized at about twenty observatories during the International Geophysical Year, I957-58.

Watts has continued the survey of the marginal zone begun at Washington in I946. About 700 photographs exposed on 400 nights have been measured with the photoelectric measuring engine. Most of the resulting profiles have been processed through the various stages ending with the derivation of a preliminary datum and about $80 \%$ through the second datum. It is expected that the operation of transferring elevations from the profiles to the chart forms will begin early in 1955 and will be finished in about eight months. The task of establishing a definitive datum will then be taken up and the charts will be completed, it is hoped, by July 1956 .

Among the subjects that it may be desirable to discuss at Dublin are: the future of the occultation programme in view of the development of the photographic method of determining the Moon's position; the choice of a satisfactory datum surface to which elevations on the Moon can be referred and the technique for defining it; applications of photography to the study of the librations and figure of the Moon.

\section{B. WatTs \\ President of the Commission}

\section{Report of meeting. 3I August I955}

President: Di C. B. Watts.

Secretary: Mrs F. M. McBain Sadler.

The Draft Report was approved without discussion.

The President called on Mrs McBain Sadler to report on the attitude of H.M. Nautical Almanac Office concerning the future of the occultation programme, in view of the development of the photographic method of determining the Moon's position. Mrs McBain Sadler described how her office had analysed the observations received in several different ways, in an attempt to find a means of reducing the work required in the occultation programme. There appears to be no way of making this reduction without a corresponding reduction in the number of observations. The only possibility indicated was the discarding of casual observations of doubtful accuracy. For the years I949-52, the following table was shown.

\begin{tabular}{|c|c|c|c|c|c|}
\hline \multirow[b]{2}{*}{ - } & \multicolumn{2}{|c|}{$\begin{array}{c}\text { All } \\
\text { observations }\end{array}$} & \multicolumn{2}{|c|}{$\begin{array}{c}\text { Retained } \\
\text { observations }\end{array}$} & \multirow[t]{2}{*}{$\begin{array}{l}\text { Percentage } \\
\text { rejected }\end{array}$} \\
\hline & $n$ & p.e. & $n$ & p.e. & \\
\hline 108 'good' stations & 5361 & $0 \div 71$ & 5168 & $0 \div 57$ & $3 \cdot 6$ \\
\hline 79 other stations & 575 & $1 \cdot 27$ & 492 & $0 \cdot 66$ & 15 \\
\hline 187 stations in all & 5936 & 0.78 & 5660 & 0.58 & $4 \cdot 6$ \\
\hline
\end{tabular}

A 'good' station was defined as one for which more than five observations had been received and for which the mean error of the retained observations was less than I".25. The 575 observations from the seventy-nine other stations take up proportionally many times as much work as those from 'good' stations; and little would be lost if they were completely omitted.

The President had supplied H.M. Nautical Almanac Office with limb corrections for three lunations in 1953. These lunations were chosen because of the extremely erratic behaviour of the lunation mean $\delta L$ and $\delta B$. The observations had been re-discussed and the results were presented in the following table. 


\begin{tabular}{|c|c|c|c|c|c|}
\hline \multirow{2}{*}{$\begin{array}{c}\text { Luna- } \\
\text { tion }\end{array}$} & \multirow{2}{*}{$\begin{array}{l}\text { Limb } \\
\text { corrections }\end{array}$} & \multicolumn{4}{|c|}{ DD } \\
\hline & & $n$ & $\delta L$ & $\delta B$ & \\
\hline \multirow[t]{2}{*}{377} & With & 61 & $+0 " 43$ & $-0 \% 95$ & \\
\hline & Without & 60 & +0.78 & $-1 \cdot 19$ & \\
\hline \multirow[t]{2}{*}{378} & With & 55 & $+0 \cdot 18$ & -0.83 & \\
\hline & Without & 54 & -0.45 & +0.25 & \\
\hline \multirow[t]{2}{*}{379} & With & 64 & +0.29 & -0.26 & \\
\hline & Without & 66 & +0.53 & $-0 \cdot 40$ & \\
\hline
\end{tabular}

\begin{tabular}{|c|c|c|c|}
\hline \multicolumn{4}{|c|}{ Al1 } \\
\hline$n$ & $\delta L$ & $\delta B$ & p.e. \\
\hline 71 & $+0 * 41$ & $-0 \div 89$ & $0 \div 29$ \\
\hline 70 & +0.64 & $-1 \cdot 11$ & $\cdot 63$ \\
\hline 63 & +0.28 & -0.85 & 0.36 \\
\hline 66 & $-0 \cdot 30$ & $+0 \cdot 27$ & .83 \\
\hline 113 & $+0 \cdot 19$ & -0.17 & $0 \cdot 34$ \\
\hline 125 & +0.31 & -0.06 & $\cdot 73$ \\
\hline
\end{tabular}

The sample is too small to lead to definite conclusions, but there can be no doubt that the application of limb corrections will give rise to a great improvement in the probable errors of the occultation results.

Mrs McBain Sadler said that her office was prepared to continue the occultation programme for the next few years, say to r962; a decision whether to continue beyond that date could be made in I958, by which time the effect of the application of limb corrections might be assessed in relation to the photographic determinations. She would like to recommend to the Commission that the occultation programme be continued without radical change; and that, while observers should continue to be encouraged to observe occultations regularly, the casual observer should be discouraged from expecting his single observations to be necessarily included in the discussions.

The Commission approved this recommendation after a discussion in which Clemence, Jackson, Hopmann, Watts, O'Keefe and Sadler took part. Sadler pointed out that it would be possible to discuss the occultation observations much more thoroughly after the application of limb corrections. Watts remarked that the photo-electric observations of disappearances and re-appearances at the dark limb would now enable dimensions of the Moon to be determined without the adverse effects of irradiation.

The President then directed the attention of the Commission to the problem of choosing a satisfactory datum surface to which elevations on the Moon can be referred, and the technique for defining it. He said that he would like to confirm that the surface which he has adopted in his work is satisfactory to users and summarized his methods.

He described how the first datum is based on circular arcs fitted to the bright limb as represented in the various photographs. In deriving the second datum, the discrepancies revealed at points common to the various profiles are used in observational equations, the solutions of which are used to adjust the radius and co-ordinates of the centre. The third and final approximation takes into account as far as possible those portions of the physical surface of the marginal zone that do not contribute to the outline of the limb. He also described the method of using libration frames to exhibit the final results.

In the discussion which followed, O'Keefe pointed out that the centre of the datum surface and the centre of gravity of the Moon would differ. Watts said that this difference could only be determined by subsequent observations and a table of corrections prepared.

J. Hopmann of the University Observatory, Vienna, reported that G. SchrutkaRechtenstamm is about to make a new reduction of the measures of the 4000 points of the lunar surface, first determined by Franz about 1895 , with the object of obtaining a better system of selenographic positions. He reported also that he had himself re-reduced the measures used by Franz in determining the figure of the Moon. (Mitteilungen der Universitäts-Sternwarte Wien, Band 6, 1952.) He considers it is desirable to take new plates with long-focus instruments for the purpose of improving these results. Schrutka's discussion of the physical libration will be published in Mitteilungen der UniversitätsSternwarte Wien.

J. Witkowski reported on the further work being carried out at the Cracow Observatory on the Moon's libration. K. Koziel has begun the reduction of the Hartwig series for the years $1877-79$; his assistant $\mathrm{J}$. Mietelski is working on the reduction of the Kazan series of T. Banachiewicz for the years I9I0-I5. Both libration series will be reduced by applying the Cracow method and will give a considerable enlargement of the observational basis for further investigation of the Moon's libration, planned at the Cracow Observatory. 
D. S. Evans drew attention to the difficulty which had been experienced in making photo-electric observations of occultations when the phenomenon was not instantaneous, although not really a grazing occultation. He had discussed this with Hirose, who said that of 420 photo-electric observations made at Tokyo Observatory five or six cases of such 'pseudo-grazing' occultations had occurred at angles of incidence of less than $60^{\circ}$. Evans considered that it would be of assistance in the interpretation of such observations to have the figure of the limb at the time of occultation or at least the rate of approach of the smooth limb. It was decided that such information could best be supplied by H.M. Nautical Almanac Office on request by observers after the observations had been made.

J. A. O'Keefe initiated a discussion, in which Atkinson, Hopmann and de GraaffHunter took part, on the errors in the adopted positions for many observatories. He pointed out that the deflection of the vertical could have considerable effect on the derived positions of the Moon. He suggested that efforts should be made to obtain observatory positions in accordance with the latest determinations of datum positions, particularly for Europe; he thought that I.G.G.U. might be asked to supply these positions.

The President informed the Commission of the result obtained from his new method of determining the mean inclination of the Moon's equator, outlined in the Draft Report. He has derived the following value:

$$
I=\mathrm{I}^{\circ} 33^{\prime} 50^{\prime \prime} \pm \mathrm{I} 9^{\prime \prime}
$$

A full account of the method will be published in the Astronomical Journal. Some discussion followed in which Weimer, Atkinson and Hopmann participated. 